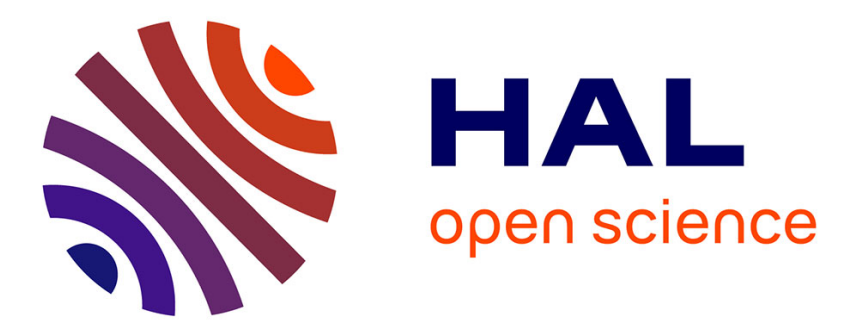

\title{
Un problème national, interallié ou international? La difficile gestion transnationale des mutilés de guerre (1917-1923)
}

Gildas Bregain

\section{> To cite this version:}

Gildas Bregain. Un problème national, interallié ou international? La difficile gestion transnationale des mutilés de guerre (1917-1923). RHPS - Revue d'histoire de la protection sociale, 2016, Grande Guerre \& protection sociale, 9, pp.110-132. 10.3917/rhps.009.0110 . halshs-01695017

\section{HAL Id: halshs-01695017 https://shs.hal.science/halshs-01695017}

Submitted on 28 Jan 2018

HAL is a multi-disciplinary open access archive for the deposit and dissemination of scientific research documents, whether they are published or not. The documents may come from teaching and research institutions in France or abroad, or from public or private research centers.
L'archive ouverte pluridisciplinaire HAL, est destinée au dépôt et à la diffusion de documents scientifiques de niveau recherche, publiés ou non, émanant des établissements d'enseignement et de recherche français ou étrangers, des laboratoires publics ou privés. 


\section{Un problème national, interallié ou international ? La difficile gestion transnationale du problème des mutilés de guerre (1917-1923)}

\section{Gildas Brégain, post-doctorant IRIS/EHESS}

\section{Résumé :}

Entre 1917 et 1923, l'échelle de résolution du problème des mutilés de guerre (nationale, interalliée, internationale) a constitué un vif sujet de débat entre médecins, dirigeants des associations de mutilés et représentants gouvernementaux et patronaux. Cet article traite de la manière dont les organisations interalliées et internationales (Comité permanent interallié pour l'étude des questions intéressant les mutilés de la guerre, BIT, Croix-Rouge) se sont saisies du problème, ont défini leurs missions et réparti leurs responsabilités. Il repose sur les archives du BIT et de la Société des Nations, ainsi que sur certaines revues associatives.

During a short period (1917-1923), the scale of resolution of the disabled ex-servicemen's problem (national, interallied, international) constitutes a subject of sharp debate between the doctors, the leaders of associations for disabled war veterans and the government's and employers' representatives. This article explains this debate at the scale of resolution of the disabled exservicemen's problem and analyses the way that the interallied and international organizations (interallied Standing Committee for the study of the questions concerning the disabled ex-servicemen, ILO, Red Cross) deal with the disabled ex-servicemen's problem, define their tasks and share their responsibilities. We use the archives of the ILO and the League of Nations, as well as some associative reviews.

\section{Mots-clés :}

handicap, mutilés, Première guerre mondiale, problème public

Disability, First world war, public problem

À l'échelle internationale, six à sept millions d'hommes se retrouvent mutilés suite à la guerre. Ils ont en majorité entre vingt et quarante ans. Cinq pays (Allemagne, France, Italie, Russie, GrandeBretagne) doivent prendre en charge plus de 800000 mutilés chacun, tandis que d'autres (Autriche, États-Unis, Pologne, Tchécoslovaquie, Royaume des Serbes, Croates, Slovènes) en ont à gérer 100 000 à 350 000. Dans tous ces pays, les mutilés de guerre se regroupent au sein d'associations aux positionnements politiques souvent antagonistes (apolitisme, communisme, catholicisme social, etc.) ; par leur grand nombre d'adhérents et le fait de représenter des victimes de la guerre, elles constituent des acteurs puissants et incontournables pour la puissance publique ${ }^{1}$.

En général, ces associations ne se rebellent pas contre les normes sociales en vigueur et mettent un point d'honneur à valoriser le sacrifice des soldats et leurs membres mutilés ${ }^{2}$. Pour résoudre le problème de leur avenir professionnel, celles de plusieurs pays européens (GrandeBretagne, Allemagne, Autriche, France, Italie) réclament que tous les employeurs publics et privés

\footnotetext{
$1 \quad$ Voir l'étude classique de Prost Antoine, Les anciens combattants et la société française, Paris, Presses de la FNSP, 1977, sur le cas de la France.

2 Gerber David (ed.), Disabled Veterans in History, University of Michigan Press, Enlarged and revised edition, 2012, p. xiii.
} 
soient contraints d'embaucher un certain pourcentage d'invalides ${ }^{3}$. Dans le même temps, les responsables politiques, confrontés à l'augmentation considérable des dépenses liées aux pensions des mutilés, sont désireux d'augmenter la main-d'œuvre disponible pour le renforcement productif de la nation. Inquiets du trouble à l'ordre social que les mutilés pourraient constituer, ils souhaitent leur assurer une vie digne grâce à une action volontariste de l'État. Les professionnels de la rééducation sont davantage préoccupés par le renforcement de leurs intérêts professionnels ${ }^{4}$. Sous la pression de ces différents acteurs, le devoir traditionnel d'assistance aux mutilés de guerre (pensions, asiles) se transforme en devoir de réinsertion professionnelle.

Plusieurs pays européens (France, Allemagne, Autriche, Italie, Pologne) adoptent ainsi entre 1916 et 1924 des mesures législatives déterminant l'obligation d'emploi des mutilés de guerre dans les entreprises publiques et privées. Ce faisant, ils mettent en place des systèmes solidaires d'organisation sociale du travail visant à garantir leur insertion professionnelle. Outre la règle du quota et l'instauration de sanctions financières pour les entreprises dérogeant à la loi, certaines mesures légales autorisent les employeurs à baisser la rémunération des mutilés de guerre au rendement inférieur. Car si les organisations d'invalides réclament généralement un salaire égal pour une durée égale de travail, les organisations patronales militent elles pour un salaire défini en fonction du rendement; et les dispositions législatives adoptées constituent souvent un compromis entre ces deux positions. La France, qui avait déjà institué par la loi du 17 avril 1916 l'accès des mutilés de guerre à des « emplois réservés » dans les administrations publiques, instaure ainsi par celle du 26 avril 1924 l'obligation d'emploi d'un quota de $10 \%$ de mutilés de guerre dans les entreprises privées ${ }^{5}$ - loi qui autorise aussi les employeurs à diminuer la rémunération en cas de diminution du rendement, tout en assurant à l'invalide la garantie d'un salaire au moins égal à la moitié du salaire normal.

L'historiographie des mobilisations associatives des mutilés de guerre et des politiques d'assistance sanitaire et de rééducation professionnelle développées après la Première Guerre mondiale date déjà de plusieurs décennies ${ }^{6}$, mais reste quantitativement faible. Si elle se renouvelle lentement

3 ILO, The Compulsory Employment of Disabled Men, Studies and Reports, Serie E, ${ }^{\circ} 2$, Geneva, avril 1921, p. 4.

$4 \quad$ Cette idée a été avancée par Diego Ortuzar dans son mémoire sur les politiques chiliennes d'assistance aux invalides, qu'il compare aux politiques d'assistance menées dans plusieurs pays d'Europe (France, Allemagne, Espagne). Ortuzar Diego, «D'indigents à contribuables ». Invalidité, médecine et travail au Chili (1916-1938), mémoire de master 2 en histoire sous la direction de Jean-Paul Zuñiga, EHESS, septembre 2011, p. 61.

Omnès Catherine, «La réinsertion professionnelle des pensionnés de guerre en France : la loi du 26 avril 1924. Un legs de la Première Guerre mondiale ? », Revue d'histoire de la protection sociale, $\mathrm{n}^{\circ}$ 8, 2015/1, p. $167-181$.

Concernant la France, Prost Antoine, Les anciens combattants et la société française, op.cit., p. 7-45 ; Montès Jean-François, 1915-1939, (re)travailler ou le retour du mutilé : une histoire de l'entre-deuxguerres, Rapport de recherche effectué pour l'Office national des anciens combattants et victimes de guerre, 1991 ; Romien Pierre, «A l'origine de la réinsertion professionnelle des personnes handicapées : la prise en charge des invalides de guerre », Revue Française des Affaires Sociales, n², 2005, p. 229-247 ; Bette Peggy, «Reclasser les victimes de la Première Guerre mondiale : le cas de la loi du 30 janvier 1923 sur les emplois réservés en France (1923-1939) », Amnis, Revue de civilisation contemporaine, n 6, 2006. 
grâce à l'adoption de nouvelles problématiques et l'utilisation de nouvelles sources ${ }^{7}$, la dimension transnationale des mobilisations associatives et des politiques publiques commence juste à faire l'objet d'études approfondies, malgré l'élan historiographique en faveur de l'histoire transnationale, croisée et connectée. Thomas Richard Davies et John Horne ont certes récemment analysé les activités des organisations internationales de vétérans durant l'entre-deux-guerres ${ }^{8}$. Antoine Prost et Jay Winter, Jean-François Montès et Bruno Cabanes ont quant à eux défriché l'action développée par la section des mutilés du Bureau international du Travail au début des années 1920 (en premier lieu les conférences d'experts convoquées ${ }^{9}$ ) ainsi que l'activité de la Conférence internationale des associations de mutilés de guerre et d'anciens combattants (CIAMAC). Leurs analyses laissent toutefois dans l'ombre deux importants acteurs, les Croix-Rouge et le Comité permanent interallié pour l'étude des questions intéressant les mutilés de la guerre (CPI). Notre article vise donc à combler cette lacune, en s'intéressant à la manière dont ces organisations internationales se saisissent du problème, définissent leurs missions et répartissent leurs responsabilités.

Le processus de transformation de la situation de trouble causée par l'existence de mutilés de guerre en problème public transnational n'est pas linéaire. L'ampleur numérique et géographique du problème ne provoque pas non plus de facto sa reconnaissance transnationale. Certains facteurs favorisent sa mise à l'agenda au sein des organisations internationales, comme la forte mobilisation des associations de mutilés de guerre et de leurs alliés (les médecins orthopédistes et traumatologues $)^{10}$, la nouveauté et l'intensité du problème, l'existence de solutions pratiques (prothèses, rééducation professionnelle) et législatives (législations nationales), l'existence de questions dont la réponse nécessite de dépasser le cadre des souverainetés nationales (tels les mutilés émigrés ou réfugiés), l'appel à l'intervention d'acteurs publics supranationaux, ou encore le désir de certains responsables gouvernementaux alliés de collaborer dans ce secteur. Mais d'autres facteurs freinent ou empêchent inversement sa transformation en problème public transnational, ainsi la noninsertion dans le champ de compétence légitime des organisations internationales, ou les stratégies diplomatiques de certains gouvernements désireux de maintenir leur souveraineté sur cette question.

Notamment Delaporte Sophie, «Le corps et la parole des mutilés de la grande guerre », Guerres mondiales et conflits contemporains, $\mathrm{n}^{\circ}$ 205, 2002/1, p. 5-14 ; Scales Rebecca, « Radio Broadcasting, Disabled Veterans, and the Politics of National Recovery in Interwar France », French Historical Studies, vol. 31, n ${ }^{\circ} 4$, 2008, p.643-678 ; Viet Vincent, La santé en guerre, 1914-1918 : une politique pionnière en univers incertain, Paris, Science Po, 2015, p. 330-388.

$8 \quad$ Davies Thomas Richard, «International Veterans' Organizations and the Promotion of Disarmament between the Two World Wars », in Eichenberg Julia et Newman John Paul (dir.), The Great War and Veterans' Internationalism, Palgrave Macmillan, 2013, p. 187-206 ; Horne John, "Beyond Cultures of Victory and Cultures of Defeat? Inter-war Veterans' Internationalism », ibid., p. 207-222.

9 Montés Jean-François, «Du mutilé de guerre à l'invalide civil; l'action du service des assurances sociales et des mutilés du Bureau international du Travail (1920-1940) », Colloque sur l'histoire de la sécurité sociale : actes du $117^{\mathrm{e}}$ congrès national des sociétés savantes, Clermont-Ferrand, 26-29 octobre 1992, vol. 117, 1994, p. 147-150 ; Prost Antoine, Winter Jay, René Cassin and Human Rights: From the Great War to the Universal Declaration, Cambridge, Cambridge University Press, 2013, p. 54-63; Cabanes Bruno, The Great War and the Origins of Humanitarianism, Cambridge, Cambridge University Press, 2014, p. 18-75.

${ }_{10}$ Hassenteufel Patrick, «L'analyse de la construction et de la mise sur agenda des problèmes publics », in Sociologie politique : l'action publique, Paris, Armand Colin, 2011, p. 46. 
Au sortir de la Première Guerre mondiale, durant une courte période (1917-1923), les échelles de résolution du problème des mutilés de guerre (nationale, interalliée et internationale) constituent dès lors un vif sujet de débat entre médecins, dirigeants des associations de mutilés et représentants gouvernementaux. La guerre provoque aussi des bouleversements contradictoires (émergence du communisme et du fascisme, aspiration au pacifisme, émergence de la puissance états-unienne, maintien de l'eurocentrisme) qui ne sont pas sans répercussions sur les choix des acteurs ${ }^{11}$.

Cet article vise donc à aborder ce débat sur l'échelle de résolution du problème des mutilés de guerre pour en éclaircir les enjeux et les aboutissements. Nous nous intéressons en particulier au positionnement et à l'action des organisations internationales, qui constituent « des espaces structurés par des relations entre des individus et des groupes d'acteurs qui, à leur tour, contribuent à leur (re)structuration $»^{12}$. Les archives de la section administrative des mutilés du Bureau international du Travail (BIT) permettent de reconstituer, outre les activités développées par les fonctionnaires du BIT, la nature des relations (coopération, division du travail, émulation, rivalité, domination, élimination, délégation) développées avec les représentants du CPI et des Croix-Rouge ${ }^{13}$. Les archives de la Société des Nations, tout comme les revues de la Croix-Rouge et du Comité Permanent interallié, complètent ces données. Ces sources ont toutefois leurs limites, ne permettant pas entièrement de faire la part des facteurs (culture organisationnelle, culture et choix des fonctionnaires internationaux, positionnement des représentants gouvernementaux, etc.) qui participent au positionnement des institutions sur ce problème.

\section{La solidarité interalliée, obstacle à l'internationalisation du problème des mutilés}

\section{L'impossible mise en place d'une conférence internationale sur les prothèses en temps de guerre}

Fondé en 1859, le Comité international de la Croix-Rouge (CICR) agit traditionnellement auprès des blessés et victimes de la guerre, mais il étend fin 1918 son action aux victimes des famines et des épidémies ${ }^{14}$. Il est dirigé par des Suisses attachés à défendre le prestige de leur pays. La League of Red Cross Societies (LRCS) est quant à elle fondée en mai 1919 par la Société américaine de la Croix-Rouge, qui souhaite orienter l'action des Croix-Rouge vers les activités médicales liées à la paix. Dans les décennies qui suivent, ces deux organismes coexistent dans le champ humanitaire ; mais le

\footnotetext{
11 Girault René, Frank Robert, Turbulente Europe et nouveaux mondes 1914-1941, Paris, Payot et Rivages, 2004, p. 159.

12 Kott Sandrine, «Les organisations internationales, terrains d'étude de la globalisation. Jalons pour une approche socio-historique », Critique internationale, $\mathrm{n}^{\circ}$ 52, 2011, p. 15-16.

Saunier Pierre-Yves, «La secrétaire générale, l'ambassadeur et le docteur. Un conte en trois épisodes pour les historiens du "monde des causes" à l'époque contemporaine, 1800-2000», Monde(s). Histoire, Espaces, Relations, $\mathrm{n}^{\circ} 1,2012$, p. 29-47.

14 Herrmann Irène, «Décrypter la concurrence humanitaire : le conflit entre Croix-Rouge(s) après 1918 », Relations internationales, $\mathrm{n}^{\circ} 151,2012 / 3$, p. 91-102.
} 
CICR reste une organisation plus puissante et géographiquement ouverte que la LRCS, qui demeure sous influence anglo-saxonne.

Dès avril 1917, le professeur florentin Hector Lévi propose au CICR de réunir la documentation existante dans les deux camps sur la rééducation professionnelle des mutilés de guerre et d'organiser une exposition internationale d'appareils de prothèse. La convocation d'une telle réunion dans un pays pacifique serait «la première manifestation internationale de retour à l'idéal chrétien de l'amour universel ${ }^{15}$. En novembre 1917, le CICR approuve l'idée d'un échange international d'informations sur les soins aux grands blessés et invalides, mais sur ce point uniquement, car d'autres sujets (organisation des ambulances, rééducation fonctionnelle) constituent des secrets de défense nationale - car pouvant contribuer à « remettre sur pied un combattant momentanément éloigné du front $»^{16}$. Le Comité international envoie en ce sens une circulaire aux sociétés nationales de la CroixRouge, mais nombre d'entre elles refusent d'échanger des informations sur les soins aux invalides, invoquant la surcharge de travail et le fait que les procédés susceptibles d'augmenter l'aptitude au travail d'invalides utilisables dans la vie civile méritent également de rester secrets. Le projet d'exposition internationale est dès lors abandonné.

En 1920, le vice-président du CICR, le Dr Frédéric Ferrière, reprend cette idée et se déclare favorable à l'organisation d'une exposition internationale de prothèses pour mutilés de guerre et du travail lors de la prochaine conférence internationale de la Croix-Rouge ${ }^{17}$. Alors qu'une solidarité interalliée prévaut dans le domaine de la rééducation, cette initiative favoriserait une entraide véritablement internationale. Mais à nouveau, le projet ne voit pas le jour: le Dr Ferrière souhaite, semble-t-il, que ce projet soit géré par les sociétés nationales de la Croix-Rouge, or celles-ci se déclarent dans l'incapacité de l'organiser.

\section{L'action pionnière du Comité permanent interallié dans la régulation de l'assistance chirurgicale et de la rééducation fonctionnelle}

À la suite d'une Conférence interalliée pour l'étude des questions intéressant les mutilés de guerre, un Comité permanent interallié pour l'étude des questions des mutilés de guerre (CPI) est finalement constitué en juillet 1917. Il est composé des délégués officiels des gouvernements alliés (Belgique, Japon, Grande-Bretagne, dominions britanniques, États-Unis, France, Italie, Portugal, Roumanie, Serbie, Russie, Grèce) mais n'a qu'un rôle consultatif. Il est dominé par les Belges et les Français, qui monopolisent la présidence et le secrétariat.

Ce comité a pour objectif de rechercher les méthodes les plus performantes de rééducation

$15 \quad$ Ferrière Frédéric, «Projet d'exposition internationale d'appareils pour la rééducation professionnelle des mutilés », Bulletin international des sociétés de la Croix-Rouge, vol. 2, n² 20, 1920, p. 890.

$16 \quad$ Ibid., p. 891.

17 Ibid. 
professionnelle, de placement et de protection sociale dans les pays alliés ${ }^{18}$. Ses ressources sont composées des subventions accordées par les États. Il centre son action sur trois éléments : l'organisation d'une conférence annuelle, la publication de la Revue interalliée pour l'étude des problèmes intéressant les mutilés de guerre, et l'organisation en 1921 d'un Institut technique et scientifique de prothèse et d'appareillage à Bruxelles. Dirigé par le chef du service orthopédique d'un hôpital situé près de Bruxelles, l'Ambulance de l'Océan, le Dr Florent Martin, cet institut est installé dans des locaux appartenant à la Croix-Rouge belge. Disposant de ressources très limitées et d'un personnel restreint, son activité se limite à la recherche de documentation sur les prothèses et à la publication de quelques études - notamment, en 1922, l'Étude sur les mécanismes de mains artificielles.

Dès octobre 1919, les dirigeants du CPI souhaitent circonscrire le rôle des Croix-Rouge à l'égard des invalides, déclarant que la question des mutilés est une question d'ordre social, économique et politique «qui doit être réservée à l'étude du Comité permanent interallié. L'action des Croix-Rouge devrait donc se borner à des interventions directes au profit des invalides $»^{19}$.

De 1917 à 1920, les délégués gouvernementaux au CPI sont en majorité des médecins et des chirurgiens réputés. Leurs débats, généralement de qualité, favorisent l'amélioration des politiques d'assistance médicale et chirurgicale et de rééducation fonctionnelle. À partir de 1920, l'existence du CPI est cependant menacée par plusieurs facteurs : d'une part, les aspects les plus urgents de la question des mutilés, à savoir les problèmes médicaux, sont désormais réglés. La protection sociale devient le problème à résoudre mais le CPI, peu compétent en la matière, n'a en outre pas la capacité de contraindre les gouvernements à adopter des dispositifs législatifs sur le sujet ${ }^{20}$. D'autre part, de nouveaux organismes intergouvernementaux (SDN, OIT) empiètent sur son champ de compétence. Plusieurs gouvernements (britannique, serbe, italien) n'accordent plus systématiquement leur confiance au CPI et font appel au BIT pour obtenir une expertise fiable sur la rééducation. Dès juin 1920, le ministre des Pensions britannique annonce son souhait de dissoudre le CPI et de confier la gestion du problème des mutilés à l'OIT et à l'Organisation d'Hygiène $(\mathrm{OH})^{21}$. À partir de 1922 , l'activité du CPI se restreint, les conférences rassemblent moins de sept délégations nationales, les débats sont moins approfondis et généralement restreints aux délégations d'invalides francophones. Les vœux adoptés sont généralement mal étudiés et plus vagues. Les réalisations du CPI se limitent désormais à des soirées mondaines ${ }^{22}$.

18 Archives de la Société des Nations, (ASDN) Genève. 1008. CPI, «Brèves indications sur la constitution et le fonctionnement du Comité permanent interallié pour les invalides de guerre », Rome.

${ }^{19}$ Ferrière Frédéric, op. cit., p. 892.

20 ASDN, 1008. Lettre, Rome, 7 février 1920, le Président du CPI au Président du Conseil des ministres, Rome.

${ }_{21}$ Revue interalliée pour l'étude des questions intéressant les mutilés de la guerre, août 1920, p. 383.

22 Cabanes Bruno, op. cit., p. 45. 


\section{La défense franco-belge de l'action interalliée dans le domaine des mutilés de guerre}

La dynamique d'internationalisation des problèmes sociaux, qui s'accélère avec la décision adoptée le 28 avril 1919 par l'Assemblée de la Conférence de la paix - de créer à Genève la Société des Nations (SDN), puis en juin 1919 avec la fin du blocus interallié (qui s'accompagne rapidement de la dissolution des organismes économiques interalliés), met en tension la dynamique de solidarité interalliée existant entre les gouvernements et entre les fédérations de mutilés. Dès le 29 avril 1919, les dirigeants du CPI cherchent à renforcer l'autorité de leur organisation tout en préservant leur champ de compétence, proposant d'admettre le CPI en tant que bureau ou commission de la SDN. La délégation italienne au CPI y est particulièrement favorable, voyant le moyen d'obtenir la protection juridique de ses très nombreux invalides émigrés et d'élargir le comité aux puissances ex-ennemies.

Le 28 juin 1920, les membres du CPI discutent âprement des modalités d'affiliation à la SDN. Alors que la délégation italienne est favorable à la transformation du CPI en comité international et à la fixation de son siège à Genève, les représentants des délégations françaises, belges et yougoslaves se prononcent contre l'internationalisation du comité. Ces derniers demandent à conserver le statut interallié et rejettent l'idée de fixer le siège du comité dans un pays neutre. Ils emportent la conviction de la majorité des membres du CPI, qui rejette l'idée d'un comité international ${ }^{23}$. Le rattachement à la SDN est accepté tant qu'il n'implique pas la modification du périmètre interallié et du siège de l'organisation.

La demande de rattachement du CPI est transmise au secrétariat de la SDN, mais elle est rejetée par ses dirigeants. Car d'une part, le périmètre interallié de l'organisation susciterait le rejet des représentants des pays neutres ou vaincus par la guerre. Selon le secrétaire général adjoint de la SDN Inazõ Nitobe, «un patronage partiel pourrait confirmer les préjugés existants désignant la SDN comme une clique de vainqueurs $»^{24}$. D'autre part, Albert Thomas, directeur du BIT, semble personnellement défavorable à la transformation du CPI en bureau de la SDN. Défendant son champ de compétence, il estime que le problème des mutilés relève du BIT et, par conséquent, que « l'organisme interallié [...] devrait, à mon sens, être fondu dans le service technique du Bureau ou être considéré comme une annexe de son service technique $»^{25}$.

Lors de la $4^{\mathrm{e}}$ conférence interalliée, en septembre 1920, le gouvernement britannique fait savoir par lettre qu'il est favorable à l'extinction du CPI : les problèmes intéressant les mutilés devraient selon lui être confiés au Bureau international du Travail et à la section d'Hygiène de la $\mathrm{SDN}^{26}$. La gestion de ces problèmes par les organismes internationaux est nécessaire pour que les pays

23 Revue interalliée pour l'étude des questions intéressant les mutilés de la guerre, août 1920, p. 382.

$24 \quad$ ASDN, 1008. Letter, October 5th 1920, I. Nitobe to Commendatore Anzilotti.

25 Archives du Bureau international du Travail (ABIT), Genève. MU 2/2/0. Note, 28 août 1920, A. Thomas à A. Tixier.

26 ABIT, MU 9.1/34. Copie d'une lettre, Genève, 2 décembre 1920, A. Thomas au directeur du Bureau de Rome. 
ex-belligerants puissent confronter leurs griefs et garantir une paix durable. Cette prise en charge par la SDN et l'OIT doit aussi permettre d'étudier le problème du reclassement des mutilés de guerre dans un cadre commun à celui des invalides de la paix, et d'appréhender ainsi de manière unifiée la question des invalides. Mais la majorité des délégués, notamment les Belges, refusent la dissolution du CPI. Un délégué français, René Cassin, propose alors une motion de compromis, conservant l'autonomie et le caractère interallié du CPI mais encourageant ce dernier à contacter la SDN et le BIT pour résoudre les questions des invalides de guerre qui sont d'ordre international. Cette motion remporte la majorité des voix, et conduit le gouvernement britannique à quitter le CPI quelques mois plus tard. À partir de septembre 1920, de fervents défenseurs de la solidarité interalliée sont désignés comme présidents du CPI : Émile Brunet, responsable socialiste et président de la Chambre des représentants de Belgique, d'octobre 1920 à octobre 1921; puis le Français Jacques Teutsch, secrétaire général de l'Union nationale des mutilés et réformés.

À partir de 1919, les dirigeants de plusieurs fédérations de mutilés de guerre de pays alliés, qui se rencontrent régulièrement aux réunions du CPI, envisagent parallèlement de s'associer. En France, les membres du conseil d'administration de l'Union fédérale des associations françaises de blessés, mutilés, réformés et anciens combattants de la Grande Guerre, de leurs veuves, orphelins et ascendants (UF) en débattent en 1919 lors du congrès de Tours. La majorité des membres est favorable à une fédération interalliée, seuls quelques socialistes et républicains privilégiant une fédération internationale. Le conseil d'administration délègue Louis Fontenaille, mutilé du Nord-Pas-de-Calais et catégoriquement opposé au ralliement avec les Allemands, comme délégué de l'UF à la quatrième conférence interalliée de Bruxelles, dont l'objectif est de discuter de la constitution d'une fédération supranationale des mutilés de guerre ${ }^{27}$. Lors de cette conférence organisée en septembre 1920, la majorité des délégués français et belges sont fortement hostiles envers l'Allemagne et n'envisagent pas de collaborer avec les vaincus. Ils dominent largement les débats. Présents à la conférence, René Cassin et Adrien Tixier tentent sans succès de convaincre les participants de la pertinence d'une fédération internationale. Il est donc décidé de constituer une fédération interalliée.

Basée à Paris, cette Fédération interalliée des invalides de guerre a pour objectif de maintenir une solidarité entre pays alliés et de défendre la cause des victimes. Elle regroupe des associations des pays alliés (Belgique, France, Italie, Portugal, Serbie), mais deux groupements importants, la British Legion et l'American Legion, refusent d'en faire partie, préférant adhérer à la Fédération interalliée des anciens combattants (FIDAC) constituée la même année (novembre 1920) et rassemblant tout à la fois mutilés et anciens combattants ${ }^{28}$. La Fédération interalliée des invalides de guerre ne déploie presque aucune activité durant les premières années - à l'inverse de la FIDAC, très active dans la défense des pensions et des droits des invalides émigrés.

À partir de 1922, la Fédération interalliée des invalides de guerre est en sursis. Peu active, 
certains dirigeants associatifs considèrent qu'elle «n'a plus la même raison d'être depuis qu'est complètement organisée la FIDAC $»^{29}$. En septembre 1922, les représentants des mutilés discutent de sa possible extinction au profit d'une adhésion de chaque association à la FIDAC ou de la création d'une Fédération internationale ${ }^{30}$. Mais la majorité des délégués belges et français continuent de privilégier une alliance interalliée et bloquent toute décision contraire. Aucune décision n'est prise.

Les délégués des gouvernements français et belge au CPI, ainsi que les dirigeants associatifs mutilés de même nationalité, refusent donc catégoriquement de s'allier avec les représentants des Empires centraux et de rendre le problème des mutilés de guerre véritablement international. Cette attitude perdure jusqu'à la disparition du CPI en 1924. Mais une dynamique d'internationalisation du problème des mutilés de guerre est parallèlement lancée dès 1920 par le service des mutilés du BIT.

\section{L'implication de l'OIT (1920-1923)}

Créée en 1919, l'Organisation internationale du Travail (dont le Bureau international du Travail est le secrétariat) a pour objectif de promouvoir, par le dialogue entre acteurs, des solutions réformistes aux problèmes sociaux afin d'éviter les conflits sociaux et économiques ${ }^{31}$. Une paix universelle et durable n'est possible que sur la base de la justice sociale. Par sa composition tripartite (employeurs, travailleurs et États), l'OIT se positionne contre le modèle d'économie étatisée mis en œuvre en URSS. Il est donc possible de la considérer comme le produit d'une première guerre froide ${ }^{32}$. Durant l'entre-deux-guerres, le BIT élabore une série de réformes sociales qui régulent les conditions du travail industriel et agricole dans le cadre du système économique capitaliste ${ }^{33}$. Ces propositions de réformes s'appuient sur les traditions socialiste réformiste et libérale sociale. Élaborées par des experts et validées par une instance intergouvernementale sous forme de conventions ou de recommandations, ces normes ont une vocation universelle. L'action du BIT vise également à démobiliser culturellement (« désarmement moral ») les peuples européens afin d'éviter une nouvelle guerre mondiale.

La légitimité de l'OIT est pourtant contestée par les organisations communistes, qui la perçoivent comme une organisation bourgeoise au service du capitalisme. Ainsi de l'Internationale des anciens combattants (IAC), créée en 1920, qui regroupe les associations d'anciens combattants socialistes révolutionnaires de cinq pays (Allemagne, France, Autriche, Angleterre, Italie). Dans la

$29 \quad$ La France mutilée, Paris, 8 avril 1923.

30 ABIT, DADG 6/12. Sixth Interralied Conference for Studying Questions Concerning the War Disabled, Lublijana, Zagreb, Belgrade, 10/19th September 1922.

$31 \quad$ Rodgers Gerry, Lee Eddy, Swepston Lee et Van Daele Jasmien, L'Organisation internationale du Travail et la quête de justice sociale 1919-2009, Genève, BIT, 2009, p. 2.

$32 \quad$ Kott Sandrine, «Par-delà la guerre froide. Les organisations internationales et les circulations EstOuest (1947-1973)», Vingtième Siècle. Revue d'histoire, n 109, 2011/1, p. 144.

$33 \quad$ Kott Sandrine, «Une "communauté épistémique" du social ? Experts de l'OIT et internationalisation des politiques sociales dans l'entre-deux-guerres », Genèses, n 71, 2008/2, p. 29. 
charte fondatrice qu'elles adoptent, ces associations s'engagent à collaborer à toute grève générale contre le régime capitaliste, à défendre les droits des victimes de la guerre, à faire une propagande pacifiste et à ne pas collaborer avec la SDN et l'OIT $^{34}$. Dirigée par son secrétaire général Henri Barbusse, l'IAC refuse par la suite toute collaboration avec l'OIT.

\section{L'affirmation de la légitimité d'une action internationale de l'OIT : la création d'un service des mutilés}

De sa propre initiative, Albert Thomas décide en juillet 1920 de créer un service chargé des questions relatives aux mutilés, en particulier invalides de guerre, au sein de la section des Assurances sociales. Un jeune instituteur français amputé du bras gauche, Adrien Tixier, est nommé en juillet 1920 à sa tête ; ancien président de la fédération départementale des mutilés de guerre du Tarn, viceprésident de l'UF, il travaille alors comme secrétaire général à l'Office départemental des pupilles de la nation du Tarn.

Le BIT considère de son ressort le problème des invalides, les mutilés étant des « travailleurs placés dans des conditions défavorables et qui, autant que les femmes et les enfants, ont besoin d'un régime de travail réellement humain $»^{35}$. Comme l'indique Adrien Tixier en 1921,

la reconnaissance nationale envers ceux qui ont souffert pour défendre leur pays a donné lieu à une appréciation plus exacte de leur droit à réparation du préjudice qu'ils avaient subi. Il s'est établi une sorte de charte des droits des invalides, variable dans la forme, mais qui comprend toujours trois articles essentiels :

1. Le droit du mutilé à une pension lui permettant de vivre convenablement ;

2. Le droit du mutilé à la prothèse et à la rééducation professionnelle qui lui permettront d'utiliser la capacité de travail qu'il possède encore ;

3. Le droit du mutilé à la protection de son placement et de son salaire ${ }^{36}$.

L'intervention de l'OIT vise donc à assurer la protection des droits professionnels des mutilés, en favorisant l'adoption d'un accord entre organisations ouvrières, organisations patronales et associations de mutilés.

Son action se justifie aussi par la gravité, la pérennité et l'ampleur du problème des mutilés de guerre, dont le nombre «dépasse certainement sept millions» dans le monde, ainsi que par l'accroissement du nombre d'accidentés du travail lié au développement du machinisme. La mise en place de politiques d'assistance est donc essentielle pour qu'ils ne deviennent pas des individus insatisfaits et révoltés. Les mutilés de guerre « représentent une force énorme que l'OIT peut utiliser pour la réalisation de son programme propre $»^{37}$, puisqu'ils militent en faveur de la paix et demandent une meilleure organisation économique du monde. Cette action est donc envisagée par le BIT comme un moyen de conquérir une légitimité internationale.

\footnotetext{
$34 \quad$ Le Combattant... Anti-Guerrier, Paris, du 25 juillet au 10 août 1920.

35 ABIT, MU 1/1/1. Texte dactylographié intitulé "Le BIT et les mutilés", non daté (postérieur à mars 1922).

36 ABIT, MU 1/1/1. BIT, "Organisation d'un service des mutilés au BIT", Genève, 28 février 1921, p. 2.

37 ABIT, MU 1/1/1. Note intitulée "L'Organisation permanente du Travail et les mutilés", non datée.
} 
Si le BIT se saisit dès 1920 de la question des mutilés de guerre, il ne s'empare réellement qu'en 1922 de celle des accidentés du travail. En février 1922, Adrien Tixier écrit à Albert Thomas pour lui demander de «confier officiellement au service des invalides ${ }^{38}$ l'étude des problèmes concernant les accidentés du travail », compte tenu du risque de voir le CPI préempter cette question ${ }^{39}$. Albert Thomas en attribue alors le traitement au service des mutilés, avec pour objectif de faire bénéficier les accidentés du travail de tous les progrès législatifs, techniques et scientifiques réalisés en faveur des mutilés de guerre.

Le champ d'action du service des mutilés résulte de négociations entre la direction du BIT, les associations de mutilés et les représentants des gouvernements rassemblés au sein du conseil d'administration du BIT, ainsi que de choix opérés par le personnel du service des mutilés lui-même, confronté à des contraintes matérielles. Le directeur du BIT fixe en 1920 à Adrien Tixier quelques priorités : l'analyse de la rééducation professionnelle, des méthodes de placement (et les règles de priorité), des salaires et des taux de pensions ${ }^{40}$.

Plusieurs organisations de mutilés suggèrent à Tixier de convoquer des conférences internationales sur les problèmes d'emploi et de prothèses. En juillet 1921, le directeur du BIT propose donc au conseil d'administration du BIT de convoquer un comité international d'experts sur les questions intéressant les mutilés (indemnisation, prothèses, rééducation professionnelle, conditions de travail et de salaire), mais nombre de membres du conseil sont réticents à attribuer des responsabilités à l'OIT sur ces sujets. Le représentant du groupe patronal, Jules Carlier, ne souhaite tout d'abord pas que la question soit réglée à l'échelle internationale, puis considère un an plus tard qu'elle « est avant tout du ressort des Croix-Rouge ${ }^{41}$. Le représentant ouvrier britannique, George Harold StuartBunning, n'est lui pas favorable à l'adoption d'une norme internationale sur le placement obligatoire qui pourrait remettre en cause la législation britannique définissant des modalités de placement, adoptée après de longues négociations avec les partenaires sociaux ${ }^{42}$. La majorité des membres du conseil d'administration, notamment les représentants patronaux et plusieurs représentants gouvernementaux, refusent quant à eux que les thématiques des pensions, du reclassement social et des salaires soient étudiées par le BIT, considérant que ces sujets revêtent un caractère national. Ils acceptent toutefois que des études soient effectuées sur la rééducation et les prothèses, à condition que ces études ne conduisent pas à l'adoption d'une recommandation ou d'une convention. Les commissions d'experts convoquées le seront donc à titre purement consultatif.

Le conseil d'administration ayant aussi demandé une liste unifiée des requêtes associatives,

\footnotetext{
38 Le service des mutilés (en anglais disablement branch) du BIT était parfois appelé le "service des invalides".

39 ABIT, MU 1/1/1. Note, 22 septembre 1922, A. Tixier à A. Thomas.

40 ABIT, MU 1/1/1. Note, 23 juin 1920, A. Thomas pour A. Tixier.

41 BIT, Procès-verbaux de la 12 e session du conseil d'administration du Bureau international du Travail, Rome, avril 1922, p. 164.

42 BIT, Procès-verbaux de la 8e session du conseil d'administration du Bureau international du Travail, Stockholm, 5-7 juillet 1921, p. 231.
} 
Adrien Tixier organise en septembre 1921 une première réunion officieuse des grandes associations de mutilés de guerre ayant déjà pris contact avec le BIT (originaires d'Allemagne, d'Autriche, de France, de Grande-Bretagne, d'Italie, de Pologne). Le BIT se contente de prêter son concours matériel (traduction et sténographie). Les délégués s'accordent sur une liste unique de vœux : le BIT est prié de réaliser en collaboration avec d'autres organisations (Organisation d'Hygiène, Croix-Rouge, CPI, etc.) des études internationales sur de multiples sujets (évaluation des infirmités et pensions, prothèses, assistance médicale, rééducation professionnelle, placement, conditions de travail); les délégués suggèrent aussi d'organiser un centre de documentation et un service de recherches scientifiques, de publier une revue, d'organiser des conférences internationales d'experts et d'adopter une convention internationale sur la protection des invalides résidant hors de leur pays. Une exposition internationale sur les prothèses de travail pourrait également être organisée.

Finalement, le conseil d'administration du BIT accepte en octobre 1921 de convoquer une conférence internationale restreinte d'experts (avec représentants associatifs et gouvernementaux) sur les prothèses. Organisée en mars 1922, cette conférence rassemble six experts désignés par les associations de mutilés (provenant de France, Grande-Bretagne, Italie, Pologne, Allemagne, Autriche), quatre autres spécialistes des prothèses et des soins médicaux, ainsi que des représentants de l'OH, de la LRCS et du CICR. Le Dr Ludwik Rajchman, directeur de la Section d'hygiène, approuve l'implication de l'OIT sur le sujet; la Section d'hygiène, qui ne revendique aucune compétence en matière de prothèse et d'orthopédie, se dit prête à offrir si nécessaire une assistance scientifique. Les experts affirment le droit des mutilés à une assistance médicale, quel que soit leur lieu de résidence. Dans un contexte où plusieurs centaines de milliers d'invalides se trouvent déplacés hors de leur pays, ils recommandent d'assurer à ces invalides émigrés le bénéfice de l'assistance médicale, de la prothèse et de l'orthopédie, via une convention internationale ou des conventions bilatérales. Par la suite, Adrien Tixier facilitera l'adoption de conventions bilatérales sur ce sujet entre la Pologne et l'Allemagne, l'Autriche et la Tchécoslovaquie, l'Allemagne et la Tchécoslovaquie.

D'autre part, les experts avalisent certains desiderata des délégués associatifs : ils demandent au BIT d'organiser un centre international de documentation, un service de recherches scientifiques et de publications, et une exposition internationale d'appareils de prothèse et d'orthopédie. Pour cela, les participants proposent au BIT d'utiliser le travail déjà accompli par le CPI et d'inscrire au budget de l'année 1923 une somme de 50000 francs pour exécuter les premiers travaux ${ }^{43}$. Ils suggèrent également de convoquer ultérieurement une autre réunion d'experts sur le travail des invalides.

Ces propositions sont étudiées par le conseil d'administration du BIT en avril et juillet 1922. $\mathrm{Au}$ sein du conseil, les représentants patronaux et certains représentants gouvernementaux sont toujours réticents à la prise en charge du problème. Après débats, le directeur propose un investissement réduit du BIT dans le domaine de la rééducation, pour sauvegarder les résultats obtenus

43 Tixier Adrien, «L'organisation de l'assistance aux invalides », Revue internationale de la Croix-Rouge, $\mathrm{n}^{\circ} 55$, juillet 1923 , p. 678 . 
dans le domaine de la prothèse et de la rééducation professionnelle ${ }^{44}$. Le conseil restreint donc considérablement le programme souhaité par les organisations de mutilés, le limitant à la seule publication d'un ouvrage sur les appareils de prothèse. Il s'oppose également à la création d'un centre international de documentation et de recherche ainsi qu'à l'organisation d'une exposition internationale de prothèses, et refuse d'attribuer le financement demandé. Dans l'impossibilité d'obtenir des fonds étatiques sur cette question, et en l'absence d'engagement ferme d'aide financière de la League of Red Cross Societies et de l'OH, les membres du conseil d'administration sont réticents à financer ce centre, craignant des dépenses élevées ${ }^{45}$. De plus, le problème des mutilés de guerre étant limité à quelques pays, la légitimité d'une action internationale se pose. Les membres du conseil acceptent toutefois de communiquer les conclusions des experts à tous les gouvernements à titre documentaire et de faciliter la conclusion d'accords bilatéraux sur la protection des invalides résidant hors de leur pays.

Quelques mois plus tard, Albert Thomas réussit à habilement manœuvrer pour obtenir l'autorisation de convoquer une autre commission restreinte d'experts sur le reclassement des invalides dans le monde du travail. Il rassure les membres du conseil d'administration en affirmant que les associations ne demandent pas l'adoption d'une réglementation internationale sur le placement des invalides et qu'il s'agit uniquement d'un examen technique ${ }^{46}$; et argumente avec succès que la convocation d'une telle réunion ne suscitera aucune dépense supplémentaire et permettra de répondre aux demandes d'assistance technique des gouvernements.

\section{Une action limitée à la diffusion de l'information et à la sensibilisation des gouvernements}

Les résultats concrets de l'action de l'OIT dans le secteur de la rééducation des mutilés restent en définitive faibles. Outre la fourniture de renseignements aux gouvernements et associations demandeuses, seuls quelques rapports sont rédigés sur le sujet et quelques conférences d'experts convoquées.

Le service des mutilés reçoit rapidement de multiples demandes d'informations sur la rééducation émanant d'organisations nationales. Pour répondre à ces requêtes, il compile une grande masse de documentation en étudiant les législations, la presse associative, les rapports parlementaires et administratifs. Dès septembre 1920, Adrien Tixier rencontre le secrétaire de la LRCS, William Rappard, pour solliciter des informations sur la rééducation des mutilés et des aveugles et l'informer de ses activités. Ce dernier demande aux sociétés nationales de la Croix-Rouge d'envoyer des documents relatifs au sujet ${ }^{47}$. Au point qu'Adrien Tixier se trouve rapidement dépassé par l'ampleur de la tâche,

$44 \quad$ BIT, Procès-verbaux de la $12^{e}$ session du conseil d'administration du Bureau international du Travail, Rome, avril 1922, p. 164.

$45 \quad$ Ibid., p. 163.

$46 \quad$ BIT, Procès-verbaux de la $17^{e}$ session du conseil d'administration du Bureau international du Travail, Genève, janvier-février 1923, p. 160.

$47 \quad$ ABIT, MU 10/4/1. Lettre, Genève, 7 octobre 1920, W. E. Rappard à A. Tixier. 
ne maîtrisant que le français et l'anglais, et peinant à lire l'allemand. Il demande donc l'apport d'un spécialiste allemand, obtenu avec l'embauche de l'Autrichien Osvald Stein, mutilé de guerre travaillant à la section des mutilés de guerre du ministère de la Prévoyance sociale à Vienne. Maîtrisant les langues slaves, Osvald Stein est chargé de dépouiller les revues de langue allemande, tchèque, polonaise, russe, et de rédiger le bulletin en allemand. Son embauche vise à favoriser les relations avec les pays ayant perdu la guerre. Adrien Tixier souhaite en effet convaincre les vétérans britanniques, belges et français de l'utilité d'échanger des informations et des expériences avec les mutilés allemands et autrichiens ${ }^{48}$. L'augmentation du personnel dédié à ce sujet au sein du BIT est d'autant plus cruciale que d'autres institutions (CPI, Fédération interalliée) disposent d'un personnel plus nombreux. Par la suite, Adrien Tixier obtient l'adjonction de M. Germanet, qui domine l'italien, le français et l'anglais. Mais bien que renforcé au plan des effectifs, le service des mutilés est doté de faibles ressources financières.

Le service publie des études sur l'indemnisation des infirmités de guerre dans plusieurs pays (France, Allemagne, Autriche, Pologne, Tchécoslovaquie, Grande-Bretagne, États-Unis) ainsi que sur l'obligation d'emploi des invalides dans les services publics et les entreprises privées (en France, Grande-Bretagne, Allemagne, Autriche, Italie). À partir de janvier 1922, le BIT publie en trois langues (français, anglais, allemand) un bulletin mensuel d'information sur les problèmes des mutilés, résumant les lois et règlements nationaux.

Les fonctionnaires du BIT convoquent aussi deux réunions d'experts internationaux, la première en mars 1922 sur l'assistance médicale et les prothèses, la seconde à l'été 1923 sur le placement et les salaires. René Cassin et Adrien Tixier choisissent ensemble les noms des délégués à ces rencontres $^{49}$. Tixier éprouve des difficultés à s'assurer la collaboration des associations de mutilés : en raison des rancœurs liées à la guerre, certaines associations des pays vainqueurs et de tendance nationaliste, comme l'Union nationale des combattants (France) et les organisations belges, sont hostiles à toute discussion avec les associations allemandes et autrichiennes. Les organisations allemandes sont, elles, longtemps méfiantes face à l'OIT, l'associant à la SDN et au Traité de Versailles. La coopération entre associations lors des réunions d'experts est toutefois facilitée par le caractère purement technique des discussions et par la neutralité du terrain (le siège du BIT est à Genève $)^{50}$.

En 1923, Adrien Tixier renforce aussi sa collaboration avec les sociétés de la Croix-Rouge. Il se rend en août 1923 à leur $11^{\mathrm{e}}$ conférence internationale pour leur exposer le travail du service des mutilés. Pessimiste sur la volonté des États de mettre en œuvre des politiques de rééducation professionnelle des invalides et conscient des limites du pouvoir de contrainte de l'OIT, il demande aux associations privées d'assistance, et notamment aux sociétés nationales de la Croix-Rouge, de

$48 \quad$ Prost Antoine, Winter Jay, op. cit., p. 54.

$49 \quad$ Ibid., p. 56.

$50 \quad$ Ibid., p. 55. 
s'impliquer (propagande, création de centres de rééducation) ${ }^{51}$. Il tente probablement par ce moyen de déléguer partiellement la gestion de ce problème, à une période où celui-ci tend à disparaître de l'agenda de l'OIT.

\section{Une collaboration difficile entre le CPI et l'OIT}

En septembre 1920, Adrien Tixier assiste à la quatrième conférence interalliée organisée par le CPI, pour réaffirmer les attributions de l'OIT en matière d'accidentés du travail. Albert Thomas lui recommande de s'y rendre avec une collection de documents, « une sorte de petit bagage établissant bien votre autorité ${ }^{52}$. Tixier y explique aux dirigeants du CPI «que le BIT ne pouvait laisser une autre organisation s'emparer d'une partie du programme qui lui a été assigné par le Traité de Paix » ${ }^{53}$. L'explication est décisive, et les dirigeants du CPI renoncent à toute implication dans le domaine.

Bien que cette conférence recommande d'entamer une négociation avec le BIT et la SDN, le secrétaire du CPI Achille Reisdorff ne prend durant les mois suivants aucune initiative en ce sens. Les dirigeants français et belges du CPI restent en effet opposés à toute collaboration avec l'OIT et la SDN. Finalement, lors d'un voyage à Bruxelles le 22 janvier 1921, Albert Thomas rencontre le président du CPI, Émile Brunet, pour discuter des modalités de collaboration entre les deux institutions. Brunet exige de conserver le statut d'organisme interallié, de contrôler le laboratoire de prothèse et de produire de la documentation législative, juridique et scientifique ${ }^{54}$. Il n'envisage ni la dissolution du CPI, ni sa fusion avec l'OIT, et ce «pour des raisons plutôt belges qu'internationales » ${ }^{55}$. Albert Thomas réaffirme de son côté les compétences exclusives du BIT concernant les mutilés du travail et argumente en faveur du traitement par le BIT de toutes les questions internationales relatives aux mutilés de guerre et du travail. Il recommande de répartir le travail de documentation sur les législations entre les deux organismes, et accepte que le CPI contrôle le laboratoire de prothèse. Il obtient du président du CPI que tout projet de convention ou recommandation concernant les mutilés soit traité par l'OIT. Les modalités de collaboration entre les deux organismes sont ensuite entérinées dans un accord officiel, ratifié par le CPI en mars 1921.

À l'automne 1921, les relations se refroidissent entre les deux organismes - le CPI étant très inquiet du programme d'action fixé par les associations de mutilés pour le BIT, très similaire au sien. De ce fait, la direction du CPI n'invite pas le BIT à se faire représenter à la $5^{\mathrm{e}}$ conférence interalliée et refuse d'envoyer un représentant à la conférence d'experts de l'OIT de mars 1922. Lors de l'assemblée du CPI, en mai 1922, Achille Reisdorff souhaite que le CPI puisse contrôler l'activité du BIT en faveur

\footnotetext{
51 Tixier Adrien, «L'organisation de l'assistance aux invalides », Revue internationale de la Croix-Rouge, $\mathrm{n}^{\circ} 55$, juillet 1923 , p. 683 .

52 ABIT, MU 2/2/0. Note, Genève, 28 août 1920, A. Thomas à A. Tixier.

53 ABIT, MU 2/2/0. Copie d'une lettre, 12 octobre 1920, A. Tixier à Gauthier.

54 ABIT, MU 2/2/0. Copie d'une lettre, 5 février 1921, A. Thomas à E. Brunet.

55 ABIT, MU 2/2/0. Note, Bruxelles, 24 janvier 1921, A. Thomas à A. Tixier.
} 
des mutilés ${ }^{56}$. A cette fin, il propose qu'un représentant du CPI soit admis dans le conseil d'administration du BIT, et inversement. Les fonctionnaires du service des mutilés rejettent de leur côté l'idée que le CPI puisse appartenir au conseil, ce qui signifierait une atteinte au principe de la représentation tripartite, selon eux ${ }^{57}$. Une représentation du CPI n'était possible que dans les commissions spécialisées organisées par le BIT, et réciproquement.

Mais tout en réaffirmant les compétences du BIT, Adrien Tixier rassure aussi les dirigeants du CPI sur la reconnaissance de leurs compétences. Les représentants des deux organismes se rencontrent à nouveau le 3 août 1922 au siège du BIT. Un accord prévoit la participation du BIT aux réunions du CPI et aux conférences interalliées, celle du CPI aux conférences internationales d'experts organisées par le BIT, et la modification du statut de l'Institut de prothèse de Bruxelles, censé devenir un organisme international placé sous la direction d'un comité composé de représentants d'organisations internationales (CPI, OIT, OH, CICR, LRCS), d'associations d'invalides et d'organismes nationaux d'assurance sociale $-\mathrm{y}$ compris des pays ex-ennemis ${ }^{58}$. Le CPI soumet l'internationalisation de l'Institut à une condition, l'appui financier du BIT. L'accord du 3 août 1922 ne délimite toutefois pas les attributions respectives de l'OIT et du CPI, Albert Thomas insistant sur le fait que « la compétence du BIT [est] fixée d'une façon pratiquement intangible par le traité de Versailles » ${ }^{59}$. De même, il n'y a pas fusion des publications des deux organismes, mais répartition du travail de documentation. Cette collaboration se traduit par l'accroissement des échanges d'informations, la participation d'un expert du CPI à la conférence d'experts de l'OIT en 1923 et la publication d'un ouvrage sur les prothèses, mais ne va pas au-delà.

Alors que le service des mutilés du BIT souhaite s'engager dans le domaine des prothèses, le champ de compétence est revendiqué depuis plusieurs années par les Croix-Rouge et le CPI. Adrien Tixier doit donc faire preuve de diplomatie. En 1923, le conseil d'administration du BIT autorise la publication d'un ouvrage sur les prothèses, mais Tixier considère que le service ne dispose ni des compétences, ni du temps nécessaires, et propose une collaboration avec l'Institut de prothèses du CPI. Le directeur de l'Institut, Florent Martin, est chargé de rédiger l'ouvrage, dont le contenu est évalué et corrigé par des experts étrangers sous la surveillance du service des mutilés. Les mutilations et les appareils de prothèse est publié par l'OIT en 1924, avec le soutien financier de la LRCS (10 000 francs) et de plusieurs associations de mutilés.

En prenant en charge la question des mutilés de guerre, le BIT s'est donc trouvé en concurrence directe avec d'autres organismes, notamment le CPI. La conquête d'une légitimité est

\footnotetext{
56 ABIT, MU 2/2/0. CPI, Assemblée plénière du 23 mai 1922. Extrait du procès-verbal publié dans "Les invalides", $\mathrm{n}^{\circ} 1,1922$.

57 ABIT, MU 2/2/0. Lettre préparée par les services du BIT en vue de la négociation du 3 août 1922 , destinée au Président du CPI.

58 ABIT, MU 2/2/0. Lettre, 22 octobre 1923, A. Tixier au Dr Carrozzi.

59 ABIT, MU 2/2/0. Protocol of the Meeting of August 3, 1922, between A. Thomas and M. Reisdorff.
} 
passée par l'embauche de spécialistes compétents, la convocation de réunions d'experts, des publications et l'aide à la création d'ONG susceptibles de soutenir son action. L'action du BIT reste toutefois faible dans ce secteur - c'est d'ailleurs le CPI qui organise l'exposition internationale sur le travail des invalides à Gand en avril 1923. Les fonctionnaires du service des mutilés ont déployé toute leur énergie pour en faire un problème international, sans complètement y réussir. Aucune solution n'a véritablement été institutionnalisée, puisqu'aucun texte réglementaire n'a été approuvé à l'échelle internationale. Les conférences d'experts convoquées par le BIT ont toutefois symboliquement légitimé certains droits pour les mutilés de guerre, très proches de ceux adoptés par les conférences interalliées.

\section{L'égalisation économique, idéal commun à la section des mutilés du BIT et aux conférences interalliées}

Outre la pension, présentée comme une légitime réparation des préjudices physiologiques et moraux subis par les invalides, les fonctionnaires du service des mutilés du BIT et les participants aux conférences interalliées réclament l'obligation légale d'emploi et la préservation du salaire intégral pour les mutilés de guerre. Les mesures qu'ils revendiquent ont pour objectif d'égaliser les conditions économiques de vie des mutilés de guerre avec celles des autres travailleurs.

\section{L'adoption de recommandations interalliées en faveur de pensions élevées et de l'obligation légale d'embauche}

Les délégués des gouvernements à la $4^{\mathrm{e}}$ conférence interalliée adoptent en septembre 1920 une série de vœux sur la protection sociale de l'invalide. Ils pointent les défaillances des législations sur les pensions adoptées dans les pays alliés, tant sur le plan de l'évaluation de la pension que des montants attribués pour chaque taux d'incapacité. L'évaluation du montant des pensions ne devrait ainsi pas se baser uniquement sur le préjudice physique, mais prendre en compte les besoins réels des invalides (chômage ou travail, besoin d'aide constante, etc.). Des sur-pensions devraient être attribuées à certains mutilés qui, « en raison de la gravité ou de la forme de leur invalidité, ne peuvent trouver un travail rémunérateur (grands invalides, mutilés implaçables...) », à ceux qui ont besoin d'une aide permanente et à «ceux dont l'appareillage est actuellement insuffisant $»^{60}$. Les pensions devraient aussi tenir compte de l'élévation constante du coût de la vie.

La conférence interalliée entérine aussi le principe d'embauche obligatoire des mutilés de guerre par les employeurs publics et privés. Ce principe doit être inséré dans la législation de chaque

60 Revue interalliée pour l'étude des questions intéressant les mutilés de la guerre, nº 5 , octobre 1920, p. 486. 
pays, et son application garantie sous peine d'amendes. Tous les employeurs privés, quelle que soit la taille de leur entreprise, «doivent être obligés d'embaucher un certain pourcentage d'hommes handicapés (disabled) capables de fournir un travail à part entière ${ }^{61}$. Les gros employeurs doivent être contraints d'employer un pourcentage d'invalides «à capacité de travail incomplète» suffisamment réduit pour éviter la désorganisation de l'industrie; mais ces invalides doivent être soumis aux mêmes conditions de travail que les valides et bénéficier d'un salaire égal. Les administrations publiques doivent elles aussi réserver un certain pourcentage d'emploi et des postes spécifiques (messagers, vigiles, etc.) aux grands mutilés. Les participants se prononcent enfin en faveur de la création d'ateliers spéciaux d'État et de colonies agricoles, ainsi que de maisons de retraites régionales pour grands invalides implaçables.

L'année suivante, les participants à la $5^{\mathrm{e}}$ conférence interalliée se prononcent en faveur d'une rééducation professionnelle d'une durée variable et sans délai de forclusion. Préoccupés par le fait que les invalides émigrés ne bénéficient généralement d'aucun droit dans leur pays d'accueil, les conférenciers exigent que les législations soient modifiées pour autoriser leur entrée et leur attribuer les mêmes droits (assurances sociales, emploi) que les autres invalides du pays ${ }^{62}$. Un accord interallié ou des accords bilatéraux devaient entériner ces principes.

\section{Les recommandations d'une commission internationale d'experts de l'OIT}

Convaincus que les invalides se trouvent dans l'impérieuse nécessité de travailler, les fonctionnaires du service des mutilés du BIT souhaitent de leur côté promouvoir leur reclassement professionnel dans les services publics et les entreprises privées. Grâce à des prothèses modernes fonctionnelles et à la rééducation, même les plus sévèrement touchés peuvent être aptes au travail. Comme les invalides, par leur rendement réduit, «sont mal armés pour affronter le jeu de la loi de l'offre et de la demande », le recours à l'obligation légale d'emploi est nécessaire. De plus, conformément aux instructions d'Albert Thomas, le service défend le paiement aux mutilés d'un salaire intégral, contre les tentatives patronales de le diminuer en fonction de la pension perçue ${ }^{63}$.

Pour légitimer cette orientation et l'ériger en norme sociale universelle ${ }^{64}$, le service des mutilés du BIT publie en 1921 un rapport sur les manières d'introduire dans les législations l'obligation légale d'emploi des mutilés de guerre, puis convoque à l'été 1923 une commission internationale technique et consultative d'experts, réunissant des représentants des associations de mutilés de guerre (majoritaires), des professionnels et des représentants ministériels de douze pays. Ces experts sont amenés à se prononcer sur un rapport préliminaire élaboré par le service des mutilés

$61 \quad$ ILO, The Compulsory Employment of Disabled Men, Geneva, Studies and Reports, Serie E $\mathrm{n}^{\circ} 2$, April 1921, p. 6.

62 Revue interalliée pour l'étude des questions intéressant les mutilés de la guerre, $\mathrm{n}^{\circ}$ 5, octobre 1921 , p. 458.

ABIT, MU 1/1/1. Note, 23 juin 1920, A. Thomas à A. Tixier.

Kott Sandrine, «Une "communauté épistémique" du social ? », op. cit., p. 33. 
du BIT, selon lequel le placement des invalides dans les services publics « ne constitue qu'une solution partielle » et qui demande également l'implication des entreprises privées ${ }^{65}$. Deux modèles sont proposés: le premier, utilisé au Royaume-Uni, fondé sur la collaboration volontaire des employeurs, qui s'engagent à occuper une proportion d'invalides dans des conditions déterminées par l'État et sous son contrôle; le second, adopté en Allemagne, Italie et Pologne, d'obligation légale d'embauche d'un pourcentage déterminé d'invalides.

Si la majorité des experts est favorable à l'obligation légale d'emploi dans les entreprises privées, le représentant du département de rééducation du ministère britannique du Travail, $\mathrm{M}$. Passmore, s'y oppose catégoriquement ${ }^{66}$. Les experts des dominions britanniques sont également réticents. Résumant la position de la majorité des experts, René Cassin juge pour sa part sévèrement le système de la collaboration volontaire, qui n'implique pas une répartition équitable de la maind'œuvre invalide parmi les employeurs, ne garantit pas l'emploi de tous les invalides et ne leur assure pas une stabilité d'emploi dans l'avenir, en particulier durant les périodes de crise économique ${ }^{67}$. Finalement, les experts convoqués par l'OIT «affirment que, pour assurer d'une manière définitive le reclassement durable des invalides dans le monde du travail, il est indispensable d'avoir recours à l'obligation légale d'emploi, en tenant compte de la diversité des conditions du travail dans les différentes nations ${ }^{68}-$ la nuance de fin de phrase étant obtenue par les experts des dominions britanniques.

Les experts s'accordent aussi pour considérer que «le montant de la pension d'invalidité ne doit avoir aucune répercussion sur le salaire », et que celui-ci doit en principe «être égal au salaire normal et courant des travailleurs valides exerçant la même profession dans la même région ${ }^{69}$. Dans certains cas exceptionnels, les invalides ayant une capacité professionnelle notablement réduite peuvent toutefois ne pas percevoir la totalité du salaire de l'ouvrier valide, si des accords professionnels homologués par des représentants des associations d'invalides l'autorisent.

Enfin, certains experts sont favorables à l'extension du bénéfice de la législation aux autres catégories d'invalides (invalides du travail, etc.), tandis que d'autres réclament la réservation du placement aux seuls mutilés de guerre. Il est finalement décidé que les législations doivent s'appliquer à tous les invalides pensionnés et que l'expérience de placement acquise puisse à l'avenir être élargie aux invalides du travail et aux autres infirmes ${ }^{70}$.

65 BIT, Le placement des invalides. Réunion d'experts pour l'étude de l'organisation du placement des invalides (Genève, 31 juillet, $I^{\text {er }}$ et 2 août 1923), Genève, BIT, 1923, p. 17.

$66 \quad$ Ibid., p. 224 ; p. 235 ; p. 261.

67 Ibid., p. 231.

68 Ibid., p. 279.

69 Ibid., p. 282.

$70 \quad$ Ibid., p. 281. 


\section{L'absence d'une convention internationale sur le travail et les pensions des invalides}

Malgré cela, aucune convention internationale n'est adoptée sur le reclassement professionnel des mutilés, alors même qu'Adrien Tixier y est, dès 1920, personnellement favorable. En novembre, il projette de constituer une commission internationale sur le reclassement économique des invalides de guerre, composée des délégués ouvriers, patronaux, gouvernementaux et des associations de mutilés. Cette commission pourrait rédiger des conventions soumises au vote de la Conférence internationale du Travail ou de l'Assemblée de la $\mathrm{SDN}^{71}$. Mais le projet échoue, les représentants gouvernementaux et patronaux du conseil d'administration du BIT refusant, dès 1921, de convoquer une commission internationale sur le sujet : nombre d'entre eux considèrent que la question du placement des invalides constitue une question éminemment nationale, et qu'elle ne concerne pas tous les États.

Adrien Tixier se rend d'ailleurs rapidement compte qu'il sera difficile d'obtenir l'adoption de mesures relatives à l'emploi obligatoire des invalides, celles-ci n'ayant été adoptées que par quelques pays européens, ayant souvent été conçues comme provisoires, et ayant souvent rencontré l'hostilité des représentants patronaux mais aussi de certains représentants ouvriers qui craignaient qu'une telle mesure nuise à l'embauche des travailleurs valides. La mesure s'oppose d'ailleurs trop frontalement à l'idéal du marché pour être inscrite dans une convention de l'OIT ${ }^{72}$. De fait, c'est souvent au nom de l'homogénéisation des conditions de concurrence entre pays que certains droits sociaux sont promus à l'échelle internationale par l'OIT.

D'autres facteurs contribuent à freiner l'adoption d'une norme internationale. D'une part, celleci pourrait impliquer, pour les Empires européens, la mise en place de politiques d'assistance dans leurs territoires coloniaux. D'autre part, le BIT ne peut bénéficier d'un fort soutien diplomatique des ONG (Fédération interalliée des invalides de guerre, IAC, FIDAC). Dès 1920, René Cassin interpelle Adrien Tixier sur la nécessité de constituer une fédération internationale des mutilés de guerre réunissant des associations des deux camps afin de surmonter les antagonismes liés à la guerre. Le fonctionnaire du BIT accepte de servir d'intermédiaire, et les réunions d'experts qu'il convoque entre 1921 et 1923 permettent de créer les premiers liens entre dirigeants associatifs français, allemands et autrichiens. En 1924, René Cassin est désigné membre de la délégation française à la SDN au titre de représentant des combattants, ce qui renforce son assise institutionnelle. À l'été 1925, les dirigeants de l'UF décident de convoquer un congrès international à Genève pour créer une fédération internationale. René Cassin sollicite alors l'aide d'Adrien Tixier et d'Osvald Stein pour organiser au plus vite ce congrès ${ }^{73}$, qui a lieu en septembre 1925 avec l'aide technique du BIT. Il conduit à la création d'une nouvelle organisation, la Conférence internationale des associations de mutilés de

\footnotetext{
71 ABIT, MU 10/1/1. Lettre, 22 novembre 1920, A. Tixier à R. Cassin.

72 Cayet Thomas et Rosental Paul-André, « Politiques sociales et marché(s). Filiations et variations d'un registre transnational d'action, du BIT des années 1920 à la construction européenne et à la Chine contemporaine », Le Mouvement social, $\mathrm{n}^{\circ} 244,2013 / 3$, p. 7.

73 ABIT, MU 16/8/1. Lettre, 16 juillet 1925, R. Cassin à A. Tixier.
} 
guerre et d'anciens combattants (CIAMAC), dont Tixier occupe le secrétariat général jusqu'au début des années 1930. Basée à Genève, cette organisation regroupe des associations provenant d'une dizaine de pays européens. Outre la défense des droits des mutilés et victimes de guerre, elle milite pour une réconciliation internationale entre pays ex-ennemis, qui pourrait garantir une paix mondiale ${ }^{74}$.

La création de la CIAMAC est néanmoins tardive : le problème des mutilés de guerre est déjà partiellement réglé par l'adoption de législations nationales, et ne figure plus à l'agenda de l'OIT. Pourtant, les dirigeants de la CIAMAC demandent en 1927 officiellement au BIT de convoquer une commission internationale d'experts pour étudier les conditions d'indemnisation des victimes de guerre. En mars 1928, Albert Thomas présente au conseil d'administration cette proposition d'expertise, mais la majorité des membres manifestent leur défiance. De fait, les représentants gouvernementaux peuvent craindre que les experts légitiment le principe d'adaptation des pensions au coût de la vie, ce qui conduirait à de nouvelles dépenses. Le président décide d'enregistrer la réponse négative du conseil, sans susciter de débats ni de vote ${ }^{75}$. Quelques années plus tard, la CIAMAC voit ses activités considérablement réduites, minée par le départ des vétérans britanniques, l'arrestation des délégués allemands par le gouvernement nazi, et le refus de participation des vétérans italiens ${ }^{76}$.

Finalement, l'action du CPI, de la section des mutilés de guerre du BIT et de la commission d'experts de l'OIT légitiment des normes de rééducation des mutilés de guerre inspirées des expériences de l'Europe continentale (obligation légale d'embauche, préservation du salaire, pension élevée proportionnelle aux besoins réels), visant à égaliser les conditions de vie des mutilés de guerre avec celles des autres travailleurs; mais ces normes «idéales» diffèrent souvent de la réalité découlant de l'application des législations nationales des pays européens (pensions modestes, capacité des infrastructures de rééducation professionnelle conséquente mais insuffisante, taux d'emploi faible).

\section{CONCLUSION}

De 1917 à 1923, plusieurs organisations internationales (CPI, BIT, CICR) ont souhaité participer à la résolution du problème des mutilés de guerre. La concurrence entre ces organismes a été particulièrement forte entre 1919 à 1922. Les dirigeants du CPI, souhaitant détenir le monopole de l'étude du problème des mutilés, ont tenté d'écarter toute implication du CICR - lequel n'a finalement eu aucune activité dans ce domaine, bien que quelques sociétés nationales de la Croix-Rouge s'y soient impliquées. Préoccupés par l'émergence d'une gestion internationale des problèmes sociaux, les dirigeants du CPI ont dès la fin avril 1919 défendu un projet de rattachement à la SDN, rejeté en 1920 par les dirigeants de la SDN du fait du périmètre interallié du comité et de l'implication récente du

\footnotetext{
74 Pour plus de précisions sur la CIAMAC, voir Prost Antoine, Winter Jay, op. cit., p. 59-63 ; Cabanes Bruno, op. cit., p. 65-70.

75 ABIT, MU 7/6/0. Copie d'une lettre, 23 mai 1928, A. Tixier à L. Viala, président de la CIAMAC.

76 Prost Antoine, Winter Jay, op. cit., p. 63.
} 
BIT. À une période de concurrence sourde entre le CPI et le service des mutilés du BIT succède ensuite une phase de négociations sur la répartition des compétences (septembre 1920-août 1922), puis une autre de collaboration infructueuse. Malgré la tendance à l'extinction des organismes interalliés à partir de 1920, les dirigeants belges du CPI se sont obstinés à faire subsister cette organisation jusqu'en 1923. Nous ignorons le poids de la pression diplomatique belge dans la fixation de cette stratégie.

Les activités déployées par le service des mutilés entre 1920 et 1923 ont tendu de leur côté à internationaliser le problème sans réellement y parvenir. Les fonctionnaires du service se sont en effet heurtés à la réticence de nombreux représentants gouvernementaux préférant conserver une totale souveraineté sur le sujet, à l'opposition des représentants patronaux craignant la légitimation de normes coercitives, à la contestation de certaines organisations de mutilés belges et françaises suivant la ligne diplomatique de leurs gouvernements respectifs, ainsi qu'à l'hostilité franche des organisations communistes. Ils ont toutefois bénéficié du soutien financier de la LRCS et de celui, symbolique, de la section d'hygiène de la SDN, pour gérer le problème des prothèses. L'action du service des mutilés reste au final d'une faible ampleur, limitée à la promotion de quelques idées (obligation légale d'embauche, préservation du salaire), sans que celles-ci soient légitimées par une recommandation officielle. Si elle s'avère un semi-échec sur le plan réglementaire, son action constitue en revanche une réussite au plan diplomatique, Adrien Tixier réussissant à faire collaborer les dirigeants associatifs français, belges, allemands et autrichiens lors de réunions techniques, dans un contexte où les diplomaties françaises et belges étaient hostiles à toute négociation avec l'Allemagne. Ce faisant, Tixier a œuvré à l'apaisement des tensions entre acteurs des pays ex-belligérants et à la préservation de la paix.

Bien que le problème des mutilés de guerre fasse l'objet de débats aux échelles interalliée et internationale, la gestion du problème a donc continué de relever de l'échelle nationale, les gouvernements désirant conserver l'entière souveraineté sur ce sujet aux lourdes implications, tant financières que politiques.

\section{Pour citer cet article :}

Gildas Brégain, «Un problème national, interallié ou international ? La difficile gestion transnationale des mutilés de guerre (1917-1923) », Revue d'histoire de la protection sociale, $\mathrm{n}^{\circ} 9,2016 / 1$, p. 110-132. 